\title{
El discurso del racismo: estructuras y estrategias discursivas $^{1}$
}

\section{Racism Discourse: Discursive Structures and Strategies}

Sandra Soler Castillo

Citation/ Para citar este Artículo: Soler, S. (2019). El discurso del racismo: estructuras y estrategias discursivas. Colomb. Appl. Linguistic. J., 21(2), pp. 194-207.

Received: 18-Sep.-2018 / Accepted: 18-Sep.-2019

DOI: https://doi.org/10.14483/22487085.13857

\section{Resumen}

Este artículo analiza el tema del racismo y su relación con el discurso. Plantea el problema de la representación del sujeto racializado, en particular el sujeto negro, a partir de la existencia de un discurso hegemónico blanco europeo que se ha ido construyendo históricamente en torno al concepto de raza, ello en una lógica de clasificación y categorización a partir de rasgos fenotípicos y características culturales que ha terminado en la muerte, la opresión y la exclusión de millones de seres humanos. El texto presentado se basa en la experiencia investigativa de la autora, con más de quince años de trabajo en el tema del racismo; también, en el trabajo de algunos de los investigadores más importantes del análisis crítico del discurso, quienes han trabajado el racismo. Se parte de la identificación de estructuras y estrategias discursivas centradas fundamentalmente en la referenciación, predicación, argumentación y el uso de recursos estilísticos y narrativos.

Palabras clave: argumentación, discurso, estrategias discursivas, estructuras discursivas, racismo

\begin{abstract}
This article analyzes the topic of racism and its relationship with discourse, and presents the problem of representation of racialized individuals, particularly black individuals from a white European hegemonic discourse that has been historically built around the concept of race. It follows a classification and categorization logic, from phenotypical and cultural characteristics, which has resulted in the death, oppression and exclusion of millions of human beings. The text presented is based on the author's research, which includes more than 15 years of experience working on the issue of racism and, additionally, collecting work from some of the most important critical discourse analysis researchers who have worked on racism. The text begins by identifying discursive structures and strategies mainly focused on referencing, predicting, reasoning, and using narrative and stylistic resources.
\end{abstract}

Keywords: reasoning, discourse, discursive structures, discursive strategies, racism

1 Este artículo reporta un avance en el área de lenguaje y comunicación a partir del proyecto: Desarrollo didáctico y tecnológico en escenarios didácticos para la formación de profesores que acogen la diversidad: factores para su implementación y su validación en la UDFJC (Universidad Distrital Francisco José de Caldas), registrado con el número (2014-0401 COLCIENCIAS), Convocatoria 661 (2014). El proyecto forma parte del Programa Arquitectura, Pedagógica, Didáctica y Tecnológica para la formación de profesores en y para la diversidad, de la alianza AIDECT-Colciencias (vigencia 2015- 2018).

2 Universidad Distrital Francisco José de Caldas, Colombia. ORCID iD https://orcid.org/0000-0003-3155-5642. ssoler73@hotmail. com, stsolerc@udistrital.edu.co 


\section{Introducción}

Según Bruner (1981, p. 23), el lenguaje es la herramienta más poderosa para organizar la experiencia y, lo que es más importante, para constituir realidades. Es lo que hace que el ser humano sea tal, pero también puede quitarle esa condición. El lenguaje tiene una doble particularidad: es un elemento constitutivo de la identidad y un medio de comunicación. Permite representar el mundo y la vida, simbolizarla y comunicarla. Pocas cosas sería la humanidad sin el lenguaje. Sin embargo, como señalara Weber (citado en Geertz, 1993, p. 20), el hombre es un animal inserto en tramas de significación que él mismo ha tejido. Construye significados sobre bases caprichosas $\mathrm{y}$ arbitrarias que se tornan en realidades y tienen consecuencias.

Hablar de raza y racismo es hablar de lenguaje y ello requiere una revisión crítica; supone pensar cómo se han construido realidades raciales a través del discurso. Este artículo analiza el problema del discurso y su relación con el racismo; plantea el problema de la representación del sujeto racializado, el cual responde a un discurso hegemónico blanco europeo que se ha ido construyendo históricamente en torno al concepto de raza, ello en una lógica de clasificación y categorización a partir de rasgos fenotípicos y características culturales.

El texto que aquí se presenta es el resultado de más de quince años de investigación de la autora en temas de racismo y discurso (Soler y Pardo, 2007; Soler, 2008; 2011; 2013; 2018), a lo que se le suma el acopio de resultados de investigación de los analistas del discurso que más han ahondado en la relación racismo y discurso; aunque resaltando que la mayor parte de los trabajos se refieren a la representación de inmigrantes, judíos o indígenas (Van Dijk, 1997; 2000, 2015; Martín y Van Dijk, 1998; Martín, 2006; Reisigl y Wodak, 2005; Wetherell y Potter, 1993; Van Leeuwen, 1996). El propósito último de este artículo es ofrecer en un solo texto a los interesados en el tema de racismo - lingüistas o investigadores provenientes de otros campos de las ciencias sociales y humanas - algunas herramientas para comprender el discurso como una práctica compleja que genera realidades, en este caso, racismo y discriminación. Tal como lo hace el análisis crítico del discurso (ACD), se apela a la identificación de estructuras y estrategias discursivas que dan cuenta de prácticas sociales. Las estructuras hacen referencia a las formas lingüísticas que posibilitan las lenguas; las estrategias, a formas sistemáticas de usar el lenguaje o, para ser más precisos, tal como las define Wodak, las estrategias responden a "un plan de prácticas más o menos preciso y más o menos intencional [...] que se adopta con el fin de alcanzar un determinado objetivo social, político, psicológico o lingüístico" (2003, p. 115).

\section{El racismo}

Sobre la idea de raza, Occidente construyó el proyecto moderno de conocimiento y de gobierno (Mignolo, 2003; Quijano, 1999). Históricamente, la emergencia de lo blanco, que dio cabida al concepto de raza como valor cohesivo y universal, si bien se remonta al nacimiento mismo de la cultura occidental con los griegos, se configura de manera particular con la llustración europea y con el discurso centrado en la racionalidad (Mbembé, 2016, p. 40). Desde entonces, como comprensión de la naturaleza humana, la razón se ató al hombre blanco y a partir de allí se establecieron jerarquías sociales en las cuales la civilización y la esclavitud fueron posibles y legítimas. Se creó una retórica de la superioridad basada en la "razón", la "verdad", el "orden" y la "normalidad", donde la mente imperó sobre el cuerpo, el intelecto sobre la experiencia y la abstracción sobre la pasión; sin embargo, esta retórica requería de otra que diera cuenta, a su vez, de la otra cara de la moneda: la invención de lo no blanco.

En principio, lo africano rápidamente se convirtió en irracional, ignorante o salvaje y se asoció con la maldad, la fealdad, el desorden la irracionalidad, la sensualidad, la superstición o la espontaneidad. De esta manera, la raza no se entiende como un hecho etéreo o teórico, sino que se constituye en un complejo sistema de gestión de la presencia de los otros, en general, y de las personas negras, en particular (Steinberg, 2005, pp. 14-16). Con la idea de raza se sentaron las bases para una empresa política que terminó legitimando a Occidente para 
esclavizar, invadir, saquear y exterminar pueblos y países enteros por más de cinco siglos.

El discurso de pretensión científica se encargó durante decenios de levantar toda suerte de teorías variopintas que hablaban de una supuesta superioridad racial de las personas blancas, de sujetos con unas capacidades intelectuales heredadas superiores, de unas mayores habilidades para las artes, la política o la filosofía; de la influencia del clima de ciertas regiones en el carácter de las personas, donde los climas europeos eran, por supuesto, los más aptos para desarrollar civilizaciones superiores. Estas ideas fueron determinantes durante décadas y constituyeron lo que se reconoce como el racismo clásico, cuyo apogeo es el texto de Gobineau (1853) quien sostenía que el factor biológico de los individuos determina su psicología y sus comportamientos morales, y que existen razas superiores a otras debido a su pureza. La raza, como asunto biológico, redujo al ser vivo y el cuerpo a una cuestión de apariencias, de piel y color. "Negro", "amarillo" y "raza" corresponden a una misma equivalencia y en ocasiones se sustituyen.

Posteriormente, bajo la influencia de las ideas evolucionistas de Darwin, se expanden por el mundo toda suerte de estrategias eugenésicas y se asiste a una obsesión generalizada por la degeneración de las razas. De esta manera, las clasificaciones raciales resultan un elemento fundamental para intentar mejorar la raza humana; países completos apuestan por crear políticas en ese sentido (Soler, 2008, p. 235). Por más de dos siglos, investigadores ofrecieron diversas clasificaciones raciales, algunas supremamente detalladas, que alcanzaron incluso las 200 razas; en el fondo de estas clasificaciones, subyacía la idea de fijar grupos homogéneos y reconocibles de tal manera que el concepto raza fuera aplicable y, así, poder establecer jerarquías entre los pueblos.

Sin embargo, en términos políticos, para autores como Mbembe (2016, p. 78), el racismo es un fenómeno que se inicia con la construcción de la idea de "negro" en el siglo XVII, con la racialización de factores centrales como el trabajo, la reducción de la movilidad de los esclavos, la prohibición del sexo interracial y el desarrollo de un sistema penal que dio origen a la esclavitud. De esta manera, se redujo el cuerpo y al ser humano a un asunto de apariencia, piel y color; una ficción en apariencia biológica que permitió a Occidente transformar el "negro" en mercancía y poner su cuerpo en la dinámica de intercambio de trabajo y de valor, una suerte de excedente de las sociedades modernas.

Durante el siglo $\mathrm{XX}$, el racismo de corte biologicista se expandió por el mundo, aunque con ciertas resistencias desde el punto de vista de la reivindicación de derechos, la igualdad y la solidaridad y también desde el campo de la ciencia. En 1962, la misma ciencia que había especulado durante casi un siglo con las clasificaciones raciales, genera un punto de quiebre a la teoría. En ese año, Frank Livingstone (1962) publica el artículo "Sobre la inexistencia de las razas humanas", en el que explica que no puede hablarse stricto sensu de razas, solo de diferentes "gradientes". Resalta que, si bien no puede decirse que todos los seres humanos sean biológicamente iguales, las diferencias no se adaptan "a los paquetes distintos llamados razas" (Livingstone, 1962). Desde entonces, la mayoría de los científicos abandonó el estudio de las clasificaciones raciales que buscaba fundamentalmente señalar diferencias y se centró en encontrar aquello que tienen en común las diversas poblaciones y los fenómenos demográficos e históricos que pudieron causar cambios fenotípicos.

Se demostró que los seres humanos difieren una media de uno por mil de su genoma, o, lo que es lo mismo, que de acuerdo con el ADN cada individuo es igual $99,9 \%$ a cualquier otro ser humano, de cualquier continente, es decir, que las diferencias corresponden al 0,1\% del total del genoma humano (Lewontin, 1972).

En el campo sociopolítico, podría decirse que el racismo del siglo XX alcanza un punto de quiebre con la caída del régimen político del apartheid en la década de 1990, el cual fue la expresión máxima del racismo blanco legitimado política y legalmente. Según Arendt (citado en Mbembe, 2016, p. 107), en Sudáfrica, Europa recurre por 
primera vez durante la modernidad a la raza como principio de cuerpo político y a la burocracia como técnica de dominación. No obstante, como ya lo han demostrado los estudios críticos en general y los estudios del discurso en particular, los discursos no desaparecen con facilidad, sino que se transforman, se sofistican, se hacen cada vez más sutiles y soterrados, de manera que es más difícil su identificación (Van Dijk, 2015). Así, aunque la raza quedó por fuera del discurso científico, no así el racismo, lo que demuestra que, como señala Aime (2016), "es el racismo el que invita a seguir teorizando sobre la idea de raza y no a la inversa... la raza no es la causa del racismo sino su pretexto, su coartada" (p. 76). En la actualidad se asiste a lo que algunos autores como Amselle (2001) y Bauman (2006) denominan nuevos racismos o ideologías de la tribu.

La globalización, los inhumanos efectos del neoliberalismo, la privatización, el veloz desarrollo de las tecnologías, su cada vez mayor auge y la amenaza de la militarización, sumada a los acontecimientos del 11 de septiembre, marcan un nuevo racismo en el que la idea de raza se desplaza del campo de lo biológico a lo simbólico, adopta otros nombres y se ancla en otros marcos de referencia que enfatizan y radicalizan los rasgos culturales o bioculturales (Oka, 2005, p. 37). Los recurridos rasgos visibles como la piel o los rasgos somáticos son ahora reemplazados por etnias, nacionalidades, civilización, religión o cultura. Sin embargo, el espíritu clasificatorio y jerarquizante sigue intacto: quién, sin importar por qué razón, tiene más o menos derecho; quién pertenece más o menos a este sitio; quién es más o menos radical a extremista, etc. En tiempos de lo políticamente correcto, los nuevos racistas se esconden y bajo el ropaje de lo cultural se convierten en defensores de las tradiciones, las lenguas, las particularidades étnicas o culturales y los derechos adquiridos como pertenecientes a un pueblo: "nuestro" pueblo.

La raza también se ha sustituido por la tradición. Esta idea supone que las sociedades tienen cimientos o raíces en el pasado, lo antiguo, y que a partir de estas avanzan hacia el futuro o lo moderno; plantea el supuesto de la existencia de una autenticidad originaria, absoluta y legítima. Sin embargo, como lo señala Aime (2016), recurrir a esta idea es una cuestión política de los tiempos actuales que trae consigo reclamos retrospectivos camuflados en función de necesidades presentes, ya sean peticiones de autonomía, intereses locales, nacionalismos, etc. En este asunto, lo que se hace es un proceso inverso a la tradición: se proyectan en el pasado imágenes o necesidades que pertenecen más al presente.

Otra manera de decir raza en términos culturales es mediante el uso de lo autóctono o la autoctonía, se trata de un mito inaugural muy potente que refiere a un "primer hombre", el autóctono, el que pertenece, el nacido allí. Como el anterior concepto, se trata evidentemente de un uso político, ya que es la base de los nacionalismos y la xenofobia. Niega la posibilidad de contacto y de construcción conjunta, representa una concepción tribal en la que el sujeto nace y no deviene, constituye los cimientos de la ideología que establece la separación entre nosotros y ellos. Bajo el amparo de una supuesta ancestralidad se proponen nuevas identidades territoriales homogéneas, fijas y cerradas. Así, como señala Aime: "La cultura es la nueva frontera sobre la que construir un nuevo relato" (2016, p. 91).

En los actuales tiempos posmodernos, en los que temas fundamentales como la solidaridad, la igualdad y los derechos de los ciudadanos se han vuelto nebulosos o han desaparecido por completo, los localismos son los nuevos criterios de juicio y legitimación. La idea de lo universal, lo social o de lo en-común cada vez se difumina más en lo particular y lo local. La igualdad social y la idea de democracia se ven resquebrajadas con el auge del capitalismo salvaje que impulsa un nuevo individualismo del "sálvese quien pueda". El descontento social producido por la globalización y la pérdida del estado de bienestar de aquellos que durante décadas se ubicaron en una situación de privilegio, en "sociedades de la abundancia", da hoy paso a los etnonacionalismos que convierten cualquier problema social en étnico.

De esta manera, la raza, con o sin variantes, continúa operando en la actualidad como principio 
de orden político. Se asiste actualmente a un nuevo racismo que, como el anterior, establece categorías y jerarquías análogas, aunque no biológicas, sino culturales o bioculturales. Sin embargo, a diferencia del racismo biológico que tendía a someter, a explotar o gobernar, este racismo cultural tiende a separar y a alejar. Bajo el principio de diferenciación, rechaza cualquier contacto con el otro racializado; no hay espacio para la puesta en común, para la interacción, para relacionarse. No hay matices, solo quedan la distinción y la exclusión. Aunque, como señala Aime (2016), en la práctica no es que existan dos formas de racismo separadas, no hay una línea temporal que las separe, ambas lógicas se mezclan, se nutren y se complementan, solo que esta última busca "cerrar el recinto", tomar distancia del otro.

\section{Racismo y discurso}

Paul Taylor (2013) señala que la raza y el racismo se fundamentan en procesos que denomina "race talk and race thinking" (p. 15). De tanto hablar de y pensar en términos de raza, esta termina convirtiéndose en una profecía y una realidad. Mediante el lenguaje se asignan significados a los cuerpos, se les atribuyen características, comportamientos, vicios $\mathrm{y}$, en ocasiones, se olvida, como señala Butler (1997, p. 11), que el lenguaje tiene una función performativa, pues algunas etiquetas no solo describen, sino que hacen cosas. Al etiquetar una realidad, esta se re-crea cada vez que se nombra; este acto lingüístico de enunciación, que en principio es individual, lleva consigo un trasfondo de base social producto del consenso social, aunque el consenso es tan solo de unos pocos que se abrogan el derecho a decidir, como ejercicio de poder.

En ese sentido, ces posible hablar de un discurso del racismo?, cंde estructuras discursivas, léxicas, sintácticas, semánticas y pragmáticas que a fuerza de repetirlas configuran el racismo como un fenómeno social? A continuación, se presentan algunos elementos discursivos que permiten hablar de un discurso del racismo.

\section{Estrategias de referenciación}

\section{La nominación: si digo negro}

Nominar es atribuir un nombre a algo. "Negro" y "raza" no han constituido nociones fijas históricamente hablando; su genealogía remite a cosas distintas, siempre inacabadas. Aunque el referente ha permanecido (el color de la piel), sus significados se han transformado a lo largo del tiempo; en ocasiones, las más, ha remitido a la diferencia, el excedente, la degradación, el embrutecimiento e incluso la inhumanidad. Piénsese, por ejemplo, para hacer referencia al discurso cotidiano, en el número de dichos y refranes que se refieren a las personas negras en términos denigrantes, "negro no la hace limpia", "negro sin amo es como hijo sin padre", "negro ni el caballo", "las negras hieden a grajo" (Soler, 2012, pp. 132-139). En otras ocasiones, las menos, ha significado esperanza, fuerza, potencia, redención. Sin embargo, "negro" ha sido una palabra que recuerda y vivifica una historia de vida desgarradora de millones de sujetos dominados bajo el concepto de raza; sujetos a los que se les ha negado la voz, el territorio, la movilidad o la libertad; sujetos que han tenido que vivir una existencia fuera de su existencia (Fanon, 2009).

El sustantivo "negro" ha hecho parte del lenguaje del insulto y del odio (Anderson y Lepore, 2013; Delgado, 1982; Butler, 1997). Como adjetivo, podría decirse que simplemente apela a una realidad perceptual; sin embargo, como nombre, en la interpelación que se hace a la persona que tiene este color de piel, desarrolla toda su fuerza performativa en tanto acto ofensivo con el poder de herir. Mediante un proceso metonímico - en la que una parte, el color de la piel, se toma por la totalidad, el sujeto- se realiza una categorización basada en la identificación física que en general remite no solo al color de la piel, sino a rasgos fenotípicos como las facciones de la cara, en especial la nariz o el tipo de cabello. Esta primera identificación gobierna la categoría y la asignación identitaria.

Identificar el asunto racial con un tema de color de la piel ha hecho que esta asignación performativa sea asumida de múltiples maneras 
por el sujeto racializado, ello en una tentativa por contrarrestar sus posibles efectos o como mecanismo de resistencia. Negro no es nadie, al menos no en cuanto a la autoidentificación, como lo discute Soler (2013) en una investigación sobre la construcción identitaria étnica de niños y niñas en contexto escolar. El estudio muestra que, al preguntar a niños y niñas por su color de piel, estos han desarrollado una escala cromática en la que distinguen entre una amplia gama de tonalidades de negro, que van desde el negro oscuro al moreno claro; así, cuanto más clara sea la tonalidad, más valoración positiva tiene. Sin duda, práctica y valoración heredadas de la época de la colonia y sus discursos, en los que el estatus social se media de acuerdo con el color de la piel.

El análisis lingüístico tiene el potencial de permitir entender cómo, usando determinadas palabras, se hacen procesos básicos de identificación y clasificación que pueden producir actos de exclusión. En las denominaciones "negro, "persona de color", "persona de raza", "afroamericano", "negrito", "moreno", "mulato", etc., hay una carga semántica que debe analizarse a la luz de las asignaciones y las autoasignaciones. Está claro que no es lo mismo que una persona sea llamada "negro", a que esa misma persona se identifique como tal, ello con todas las críticas y discusiones que este hecho puede conllevar.

La semántica de los nombres se complejiza allí donde hay de por medio relaciones asimétricas de poder; por eso, sería mejor especificar rasgos identitarios como el color de la piel solo donde sea necesario. Como señala Faloppa (2016, p. 137), con esto no se trata de esconder una característica física, solo de no marcarla cuando no hace falta. De lo contrario, por qué no se llaman blancos a las personas blancas y se empieza a distinguir entre la variedad de tonos de piel blancos, como bien lo resaltan los niños afrodescendientes cuando reflexionan sobre los colores de la piel y sus asignaciones (Soler, 2013).

Reducir el problema del racismo a las denominaciones léxicas posibles o deseables puede desviar el problema de suverdaderotrasfondo: pensar de manera errada que las categorías expresadas por los nombres son naturales e inevitables en lugar de sociales, históricas y circunstanciales. La raíz del problema no es cuál de los nombres es mejor, más aceptable o más políticamente correcto, sino por qué se nombra y por qué se hace como se hace (Faloppa, 2016, p. 137).

\section{El uso de pronombres: nosotros versus ellos}

La retórica de la exclusión y el racismo se expresa lingüísticamente en las personas del discurso. El racismo ha desarrollado una lógica en la que la identidad se ha reducido a la existencia de un "yo" y a la negación de cualquier "tú" (Van Dijk, 1998; Butler, 2016).

Las personas "yo/nosotros", de un lado, y "tú/ellos", de otro, expresan lingüísticamente el resultado de procesos de identificación y diferenciación que responden a una ideología de dominación de unos, los blancos, sobre otros, los racializados, en particular las personas negras. Los que comparten algunos elementos pertenecen y conforman un "nosotros", son semejantes, poseen unos rasgos comunes, comparten unos valores; los otros, "ellos", son diferentes, no tienen nada en común con "nosotros", no comparten ciertos valores. "Nosotros" es la medida del bienestar, "ellos" lo amenazan; en ese sentido, "nosotros" es el portador de rasgos positivos, "ellos" está asociado con lo negativo. Como lo resalta Van Dijk (2000), nosotros/positivo y ellos/negativo constituye el cuadrado ideológico que conforma las relaciones de poder y dominación en las sociedades actuales.

Este uso discursivo de los pronombres personales se ha acentuado mediante otra partícula lingüística que busca reiterar esta lógica diferencialista. Mediante el uso de los posesivos de la primera persona singular y la primera persona del plural en masculino y femenino "nuestro", "nuestra", "nuestros", "nuestras", se ha creado una retórica de la reiteración que tiende a afianzar aún más las diferencias. Son de uso frecuente enunciados del tipo: "ellos no respetan nuestras costumbres", "ellos amenazan nuestra seguridad", "ellos abusan de nuestras instituciones" (Van Dijk, 1993; 1998). 


\section{Estrategias de predicación}

\section{La adjetivación: las cualidades que se atribuyen al Otro}

Una vez asignado un nombre al otro, se procede a la atribución de cualidades que se supone le pertenecen o se corresponden con dicho nombre, cualidades que contribuyen a caracterizarlo y comprenderlo mejor. Este proceso se realiza mediante juicios de valor establecidos por el sujeto que asigna la calificación, juicios que, en el caso del racismo, no se hacen atendiendo a la objetividad, sino a prejuicios y estereotipos. De esta manera, el nombre "negro" se acompaña de adjetivos mayoritariamente negativos que aluden, por una parte, a carencias y, por otra, a excedentes. Carece de humanidad, por lo que es inhumano; carece de civilidad, por lo que es bárbaro; carece de razón, por lo que es irracional; carece de inteligencia, por lo que es ignorante; carece de valor, por lo que es pobre; carece de moral, por lo que es inmoral, etc. Excede en altivez, por lo que es soberbio; excede en la acción, por lo que es apasionado; excede en el ánimo, por lo que es depresivo y nostálgico, etc.

"iSucio negro!", de esta manera inicia Franz Fanon (2009) su autorrelato de reconocimiento de persona negra a través de la apelación y la mirada del sujeto blanco, y continúa: “...el negro es una bestia, el negro es malo, el negro tiene malas intenciones, el negro es feo, mira, un negro" (p. 111). Obsérvese cómo, a la ya marcada nominación, se agregan cualidades referentes a las supuestas características y costumbres de las personas negras, las cuales terminan por completar el círculo semántico negativo de representación. En ese mismo sentido, Soler (2012, p. 135) señala cómo en el refranero colombiano existen variadas expresiones para referirse a este hecho; así, dichos como "las negras hieden a grajo" son frecuentes. Incluso, resalta que un escritor como Gabriel García Márquez, en El amor en los tiempos del cólera, hace eco a este prejuicio al poner en boca del personaje femenino central estas palabras para referirse a la amante de su esposo: "Y lo peor de todo, carajo: con una negra. Él corrigió, mulata. [...] —Es la misma vaina, dijo, y solo ahora lo entiendo: era un olor de negra" (p. 135).
En términos generales, en el proceso de representación del otro, el otro racializado se le asigna lo que podría llamarse el lenguaje del mal: mal hablado, mal vestido, mal comportado, mal construido y, por tanto, mal ciudadano, mal estudiante, mal parecido, etc., de manera que se devalúa su lengua, sus costumbres, sus valores, sus capacidades, etc.

\section{La predicación}

La predicación tiene que ver con el tipo de acciones que se atribuyen a los sujetos y sus rasgos semánticos, positivos o negativos. En el caso del discurso racista, los otros son, la mayor parte de las veces, agentes de acciones semánticamente negativas; así, por ejemplo, Vargas (2016), al analizar las crónicas de Indias, señala cómo históricamente, durante la conquista y la colonia de América, se predicaron acciones de la alteridad indígena, tales como asesinar, matar, mentir, comer carne humana, atacar, herir, etc., Posteriormente, en épocas más recientes, al otro se le atribuyen acciones como ilegal, desorden público, incendio, destrucción, alteración del orden público, actitud amenazante, etc. (Van Dijk, 1998, p. 201). De esta manera, se construyen discursos como: "Ellos vienen y nos quitan nuestros trabajos, invaden nuestros espacios, colapsan los servicios sociales, se aprovechan de nuestros recursos, violan nuestras mujeres, amenazan nuestras vidas, etc.".

Martín (2006), al analizar la representación de los inmigrantes en los medios de comunicación y el discurso político, identifica tres tipos principales de acciones atribuidas a los extranjeros: agentes de procesos negativos con acciones violentas y reprobables como irrumpir, causar lesiones, robar o asaltar; agentes pasivos a la espera de beneficios por parte del Estado, mediante verbos como ayudados, atendidos, integrados; y como agentes experimentantes de situaciones de las que no son responsables ni pueden incidir, con expresiones del tipo "viven en condiciones de hacinamiento", "padecen situaciones injustas".

Esta representación de las acciones negativas del otro, especialmente expresadas en el discurso 
político y de los medios de comunicación, construye, por una parte, una imagen negativa del otro como un sujeto activo en cuanto individuo abusador y peligroso y, por otra, como sujeto pasivo que arriba a espacios que no le corresponden, que no son suyos, en busca de beneficios: los sistemas de educación, de salud y de bienestar en términos generales (Welch, 2013; Van Dijk, 2015; Howard, 2002). Pocas veces se escuchan o leen discursos que resalten las acciones positivas de la alteridad o el exogrupo y las pocas veces que se hace es para reforzar estereotipos como que los "negros" son buenos para el deporte o para la música (Soler, 2009, p. 19).

\section{Estrategias argumentativas. Retóricas del} discurso racista: ¿por qué digo lo que digo?

El discurso racista se justifica de diversas maneras, mediante diversas retóricas (Reisigl y Wodak, 2005; Wetherell y Potter 1993) y, como ya se ha mencionado, se transforma constantemente en la búsqueda de nuevos argumentos una vez su propia carencia de lógica los rebate. Debido a los alcances del artículo, no se plantea un análisis argumentativo del discurso racista como tal; sin embargo, es importante identificar algunos argumentos que sustentan tal discurso.

\section{Retórica 1. Autorreferencialidad: yo superior, yo ideal de humanidad}

La autorreferencialidad fue el punto de partida para entender el mundo en Occidente y las relaciones con los otros y con el paso del tiempo ha permanecido constante en el discurso racista. Un "yo" que se contempla en el espejo y que es incapaz de mirar a otra parte, porque solo se ve a sí mismo; lo demás, lo otro, no constituye otra cosa que un reflejo empobrecido del ideal de hombre que él representa: el blanco. El otro constituye un prototipo de una figura prehumana incapaz de liberarse de la condición de animalidad. La relación con el otro, entonces, solo subraya y enfatiza una ausencia: la usencia de lo mismo, de la condición humana. Por más que el otro se esfuerce, hay un problema ontológico de fondo, no se pertenece a la misma especie.
Retórica 2. Extrañamiento: tu alteridad física y moral

La primera lógica conduce de manera directa al extrañamiento. Si el otro no es mi semejante, ¿quién es?, ¿por qué está aquí? Puestos en la necesidad de justificar la presencia del otro, este se constituye en una presencia extraña. Es lo opuesto al deseo, al ideal. En consecuencia, si no es idéntico, debe ser anormal, le falta algo: es el reflejo de una carencia. En un principio, la carencia se identificó como la condición de humanidad: el otro no es humano. Luego, fue la razón: el otro no es racional. Después, fue la falta de valores: el otro es inmoral. El otro nunca será un ser completo desde la perspectiva del "yo", nunca podrá ser como "yo"; siempre será un extraño y como extraño, indeseable. Tampoco interesa conocerlo, no se quiere saber nada de él ni de sus costumbres. De esta manera, se cae en la lógica perversa de "no me gusta porque no lo conozco" y "no lo conozco porque no me gusta".

Sin embargo, el otro es útil para afirmar la identidad propia: el lugar de sí en el mundo. Justifica mis instituciones, mis leyes, mi ética, mis discursos. En esta lógica binaria, la presencia del otro ratifica mi condición de normal, mi rectitud, mi inteligencia. El "yo" expulsa sus contradicciones, sus miedos, sus odios, sus irracionalidades y los deposita en el otro, constituyéndolo de esta manera en la antítesis de su identidad. Sin esta posible comparación no sería posible justificar la existencia del mal en el mundo. Frente a lo malo, no se es el culpable, la culpa es del otro, quien sintetiza toda la posibilidad del mal. El otro permite nombrar el mal, la barbarie, la herejía, la enfermedad, la mendicidad. De manera que, como señalan Larrosa y Pérez de Lara (1998):

La alteridad del otro permanece como reabsorbida en nuestra identidad y la refuerza todavía más; la hace, si es posible, más arrogante, más segura y más satisfecha de sí misma. A partir de este punto de vista, el loco confirma nuestra razón; el niño, nuestra madurez; el salvaje, nuestra civilización; el marginado, nuestra integración; el extranjero, nuestro país; el deficiente, nuestra normalidad. (Citado en Skliar y Duchatzky, 2015) 
Esta retórica implicó que se ejercieran ciertas políticas sobre los sujetos de raza con exterioridad salvaje o no civilizada, políticas conducentes en primera instancia a su desaparición física y luego a su corrección y rectificación. Se ensayaron distintos tipos de dispositivos; sin embargo, a pesar de la violencia que se ejerció históricamente contra los cuerpos diferentes, cabía la posibilidad de un encuentro, así fuera en términos de ejercicio de poder: lo educo, lo trasformo, lo civilizo, lo asimilo, lo integro. En últimas, le reduzco su diferencia. De esta manera, la alteridad, si quiere formar parte de la totalidad, debe apartarse de sus marcas identitarias: debe desracializarse, desexualizarse, desprenderse de todo aquello que lo identifica como otro.

Retórica 3. Amenaza: tu diferencia cultural, tu peligro

En la actualidad, ante el desmonte de las teorías biologicistas de las especies humanas y de las clasificaciones raciales, los argumentos racistas se han transformado y adquieren una nueva lógica, quizás más extrema, si aún es posible. La raza y otras formas de diferencia se convirtieron en el lenguaje privilegiado del odio social; la raza dejó de tener un significante casi exclusivo en el color de la piel y ahora agrupa otro orden de categorías, no biológicas, sino culturales como la lengua, la religión y las costumbres que convirtieron a los diferentes en los nuevos enemigos. Los otros continúan teniendo alteridades físicas y morales diferentes, pero, además, representan la inminencia de una amenaza para la seguridad y el bienestar de la población blanca (Enck-Wanzer, 2011).

Esta lógica sumativa de las diferencias y las consecuencias exacerba el odio hacia los otros y niega toda posibilidad de encuentro. Se trata ahora de mantenerlos lejos, aislados, fuera de nuestra mirada y alcance. Bajo la dinámica de riesgo/protección, la población blanca ha generado miedo en el grupo y, a la vez, se ha instalado como la solución: "yo te protegeré". De esta manera, han aparecido figuras mesiánicas con esta función. Esta retórica también ha dado paso a nacionalismos extremos en casi todos los países del denominado primer mundo, los cuales logran invisibilizar en sus ciudadanos el verdadero problema: un modelo económico neoliberal que arrasa con el estado de bienestar de la población, no solo en los países emergentes, como venía sucediendo históricamente, sino también en los países del primer mundo (Giroux, 2013).

Para sintetizar, el racismo ha tenido un centro unificador que ha justificado históricamente la exclusión, la diferencia. Justificación que partió de un principio de identificación y diferenciación en el que las personas negras constituían una realidad aparte, por fuera del espíritu y el proyecto universal de la humanidad. Luego, la diferencia se relativizó un poco y, ante la duda de una posible humanidad, el ser aparte se convirtió en un ser por transformar: quizás sí posea algo y de lo que se trata es rescatarlo y transformarlo. Por último, las diferencias se radicalizaron, se ampliaron y ya no es posible la comunión con ningún otro; es necesario alejar dichas diferencias que se han convertido en un peligro para ese "yo" siempre temeroso de lo diferente.

\section{Estrategias estilísticas. Concesión aparente}

La sintaxis y algunos aspectos estilísticos también forman parte de la manera como se ha referido discursivamente al otro racializado. La concesión aparente es un mecanismo mediante el cual se intenta que las ideas, las acciones o las decisiones frente al otro parezcan menos duras o fuertes de lo que realmente son; asumen formas de lo denominado "políticamente correcto" que incluye la no afirmación directa, sino su expresión mediante rodeos que permiten ocultar y disminuir la fuerza de las afirmaciones. Incluso pretenden crear un efecto contrario, es decir, demostrar que podrían beneficiar al otro.

La concesión aparente, en general, se expresa lingüísticamente mediante la forma: "Yo no soy racista, pero...". La primera parte de la oración afirma un hecho, pero acto seguido, mediante la conjunción adversativa "pero", introduce el verdadero argumento o la idea que se quiere defender: "Yo no soy racista, pero estoy cansado de que ellos vengan aquí a usurpar nuestros derechos". Nótese cómo la afirmación inicial pierde todo su valor, da la impresión de un acuerdo, pero en realidad termina por ratificar el primer elemento, es decir, "yo sí soy racista". Se trata de un atenuador 
discursivo que en apariencia hace una salvedad, pero que termina por ratificarla.

Este tipo de discurso, también denominado empatía aparente (Van Dijk, 1998; 2000), puede introducirse, con el mismo sentido, sin la presencia de la conjunción adversativa "pero". De esta manera, en la primera parte del discurso se introduce una idea acompañada de un elemento negativo que resulta difícil de debatir para, posteriormente, presentar la idea fuerte, en general, una acción que desacredita al otro o que intenta limitarlo. Un ejemplo de este tipo de argumentación sería: "No queremos ejercer nuestro derecho a defendernos; sin embargo, resulta preocupante la actual situación de violencia en el país por la llegada de tantos inmigrantes....". La primera frase del enunciado niega una acción que, sin embargo, mediante un argumento contundente, se hace casi inevitable.

\section{Metáforas del discurso racista}

La expresión del racismo mediante metáforas es un recurso estilístico recurrente en el discurso racista (Reisilg y Wodak, 2005; Cisneros 2008). El racismo, al ser una teoría fuertemente sustentada en el discurso científico, utiliza la metáfora como instrumento para su comprensión. Estas figuras retóricas intentan explicar ideas con alguna complejidad mediante su sustitución por conceptos más fáciles de entender, aquellos con los que el individuo está más familiarizado por su experiencia cotidiana.

La idea de pureza de las razas trajo consigo la metáfora ambientalista de la "contaminación"; esta metáfora de corte ontológico, siguiendo la clasificación de Lakoff y Johnson (1991), supone la comparación entre dos dominios de conocimiento. De un lado, el individuo, la sociedad o la cultura como espacios o ambientes cerrados, limpios y puros; del otro, factores ambientales externos contaminantes. En el contacto, la mezcla, los primeros pueden sufrir procesos de contaminación producto de los ambientes externos sucios. Ideas que se expresaron en frases como "la contaminación de la sangre", "la contaminación de los pueblos", o "la contaminación de las costumbres", y que enmascaran una lógica etnocéntrica, esencialista, que defiende la idea de una supuesta consistencia interna del grupo dominante.
En esa misma lógica, el discurso racista también emplea metáforas ontológicas de la medicina, en particular del campo de las enfermedades, como el "contagio" (Reisilg y Wodak, 2005). Basa su lógica en la existencia de cuerpos sanos y cuerpos enfermos. Cuerpos sanos que en contacto con cuerpos enfermos pueden "contagiarse", de modo que los vicios de los cuerpos racializados, enfermedades, pueden contagiar los cuerpos sanos de las personas blancas o con cuerpos no racializados. Se expresa en frases coloquiales como "Aléjate de mí, me puedes contagiar con tu pereza". El racismo también se ha expresado en términos médicos, de "epidemias" o "brotes"; así, de la misma manera que hay brotes de varicela en la población, también puede haber "brotes de racismo", aspectos que convierten el racismo en un fenómeno biológico y no social o cultural y que da lugar a su naturalización.

El paso de la comprensión de la raza en relación con las culturas trajo consigo metáforas espaciales y biológicas. Las culturas se percibieron como "recintos", como espacios cerrados en los que no había lugar para elementos externos. Esta metáfora se reforzó con la idea de los individuos como "árboles" y las costumbres como "raíces", y se evidencian en expresiones metafóricas como "yo soy de aquí porque mis raíces están aquí". En oposición a aquellos quienes no pertenecen aquí, y que se evidencia en frases como "Esta no es tu tierra".

En la actualidad, una de las metáforas más empleadas, en especial por los medios de comunicación, es la metáfora de "las oleadas", así lo han destacado autores como Van Dijk (2000). Esta metáfora proviene del campo de los fenómenos marítimos y denota la llegada de agentes externos a un lugar - en general, un país o ciudad-. El arribo no necesariamente es por vía marítima; sin embargo, se habla de "oleadas" para referirse a la cantidad de personas y a la fuerza destructiva. En los periódicos son frecuentes titulares como "oleadas de inmigrantes arribaron a las costas españolas". Esta representación tiene varios efectos en el lector u oyente, pero alude primeramente al sentimiento de miedo. Una oleada no es algo positivo, es un fenómeno natural que causa daños. 


\section{La narración}

La narración es, como señalara Ricoeur (1995), una reconstrucción particular de la experiencia, realizada mediante un proceso reflexivo en el que se da significado a lo sucedido y vivido. La narración cumple variadas funciones socioculturales que incluyen entretener, persuadir al interlocutor $\mathrm{O}$ contribuir a la reproducción de normas, creencias, valores o ideologías.

En términos generales, las historias sobre grupos racializados funcionan como quejas 0 como expresiones de experiencias o prejuicios negativos (Van Dijk, 1997). Más que narrar, estas historias sirven como argumentos o pruebas de ideas más generales. Las historias dan cuenta de acontecimientos que resultan relevantes para el narrador y que, en principio, se suponen "dignos de contar". Sin embargo, la narración de encuentros entre grupos interétnicos no siempre responde a esta lógica. Trata sobre encuentros y relaciones interétnicas en las que solo el simple hecho del contacto o el encuentro es ya narrable, lo que supone una extrañeza, es decir, la creencia de que no es normal, frecuente o corriente que se den encuentros interétnicos en los grupos sociales.

Sin embargo, más allá del encuentro, también es importante la naturaleza de las acciones y el tipo de sucesos que se narran, y que el narrador, blanco o mestizo, en general, considera diferentes a los de los miembros de su grupo. Dicha diferencia es valorada en la narración de manera negativa. Siguiendo la estructura narrativa propuesta por Labov y Waletsky (1967), los acontecimientos narrados en la acción complicante suponen hechos negativos que inmediatamente son evaluados por el narrador como contrarios a las normas y valores de la sociedad dominante. De manera que son frecuentes historias de violencia, robos, ataques, violaciones, etc., las cosas y las acciones positivas de los grupos racializados pocas veces se narran, lo que supone que las historias son ejemplos de estereotipos y prejuicios étnicos y raciales (Van Dijk, 1997).

Existe una creencia prejuiciosa respecto a que los grupos racializados, en especial los afrodescendientes, son más violentos o delinquen más que las personas blancas. Esta creencia hace que sus acciones sean narrables o noticiables, en el caso de las historias contadas por los medios de comunicación. Si las personas blancas cometen los mismos crímenes, estos no son noticiables. De esta manera, por ejemplo, si un mismo día ocurren varias violaciones a mujeres, cometidas por personas blancas 0 negras, solo serán noticia aquellas cometidas por los sujetos negros, tal como lo analizara Polletta (2006) al referirse a la manera como fue tratado por los medios de comunicación en 1989 el caso de la violación de una joven que trotaba en el Central Park y supuestamente fue violada por un grupo de jóvenes negros. Los medios priorizaron la narrativa dominante de la mujer blanca victimizada por hombres negros victimarios, a pesar de que ese mismo día sucedieron otros raptos con violadores blancos. Los medios evitaron contar narrativas alternativas que hicieran la información más imparcial (Polletta, 2006, p. 16). De lo anterior se desprende que lo que finalmente refuerza la naturaleza de la historia y de las acciones que se narran es que estas deben ser interpretadas como amenazas para el grupo dominante, son historias en las que los blancos son víctimas de los grupos racializados, de allí que sean relatos de delitos o violencia en los que la seguridad de los blancos se ve amenazada.

Otra estrategia narrativa comúnmente usada y escuchada en las narrativas es la negación o el alegato de su desconocimiento. Los narradores se excusan bajo el supuesto desconocimiento, rehusando la responsabilidad de sus ofensas, la cual ha llevado incluso a autores como Mills (citado en Jungkunz y White, 2013) a hablar de una "epistemología de la ignorancia" y de la necesidad de trabajar en la responsabilidad racial.

\section{Conclusiones}

La representación del otro ha estado marcada históricamente por la violencia discursiva. Mediante el discurso se ha condicionado cómo pensary quépensar del otro. El poder del lenguaje radica en su capacidad para crear realidades; de esta manera, conceptos como raza y racismo se convirtieron en algo más que palabras, en realidades. En la actualidad, si bien hace ya más de un siglo se cuestionó científicamente 
la idea de raza, esta continúa siendo parte del léxico habitual de los medios de comunicación, la política o la escuela. Una vez creada la realidad, su vuelta atrás resulta problemática, sobre todo cuando su correlato de acción permanece totalmente vigente, es decir, el racismo.

El racismo está acompañado de una serie de palabras que contribuyen a construir su marco de acción, de donde se resalta el carácter performativo del lenguaje y su capacidad para hacer cosas - herir, en el caso del racismo-. Aunque es importante resaltar que las palabras en sí no son problemáticas, lo es su capacidad para inferiorizar al otro; así, expresiones como "negro", "negrito", "mi negro" provocan la reacción en los sujetos interpelados con estos nombres para indicar su poca asertividad: "No soy negro, como tú no eres blanco". Más allá de las palabras está su función discursiva y retórica, su uso apodíctico y terminante.

El problema de la denominación mediante determinados nombres implica entonces entender que las categorías elegidas para representar al otro no son naturales, neutras o inevitables; supone, en cambio, reconocer que se trata de hechos sociales e históricos. En la actualidad, parece que el problema estuviera en seleccionar las palabras, las políticamente más correctas; sin embargo, esto deja de lado el verdadero problema: ¿por qué se nombra?, ¿por qué se hace como se hace?

Diversas justificaciones se dan al nombrar la diferencia, la diferencia racial en particular. Así, podría identificarse una línea retórica que comienza por la autorreferencialidad en la que el sujeto "yo" se erige como medida de todas las cosas. Pasa en seguida al extrañamiento del otro que tiene en frente. Si el otro no es igual a mí, entonces es diferente, no es otro "yo", sino un anormal, o desviado. Por último, y, en consecuencia, se crea la retórica de la amenaza. El otro está aquí para quitarme mis pertenencias, es violento, amenaza incluso la existencia propia. Retórica que en un primer momento incluyó las diferencias, luego pasó a la asimilación para terminar, finalmente, en la declaración de imposibilidad total de contacto.

Las lenguas ofrecen a los hablantes diversidad de posibilidades de significación y expresión. Los sujetos eligen, de una amplia gama, aquellas que mejor responden a sus necesidades e intenciones. La sintaxis ofrece posibilidades de ordenamiento de las oraciones para atenuar o enfatizar el pensamiento; la concesión aparente es una forma recurrente que permite "cuidar nuestra cara" o imagen del hablante frente a los demás. Dado que no es políticamente correcto ser racista, organizo los enunciados de manera que escondan los prejuicios racistas.

De igual manera, estilísticamente, el hablante puede recurrir al uso de lenguaje figurado para representar la realidad; la metáfora es un recurso recurrente que ayuda a la comprensión de los fenómenos. El racismo ha adoptado metáforas de diversos campos y ha construido una imagen del sujeto racializado en términos negativos, como plagas, epidemias o enfermedades.

La narrativa también es un tipo de discurso que se organiza para contar la percepción del otro a través de una propia experiencia o la de terceros; así, con frecuencia aparecen en el discurso relatos del otro centrados en supuestas acciones negativas, las cuales terminan convirtiéndose en hechos noticiables y contribuyen a crear una imagen negativa del otro, en especial, el sujeto negro.

Frente al cambio en los discursos y las retóricas, se hace cada vez más necesario reconocer que el discurso es más que una práctica simbólica o un modo de comunicación, es una práctica que produce efectos. De allí la necesidad de seguir develando las lógicas del discurso racista, sus constantes y transformaciones para comprender cómo opera el racismo producto del cambio de la naturaleza de los discursos, pero también para ofrecer posibilidades y nuevas maneras de representación que reten el statu quo y terminen de una vez con todas las formas de discriminación y racismo.

\section{Referencias}

Aime, M. (2016). Se dice cultura se piensa raza. En M. Aime, (Ed.), Contra el racismo (pp. 73-104). Barcelona: Editorial Libros.

Amselle, J. (2001). Branchement: Anthropologie de l'universalité des cultures. Paris: Flammarion. 
Anderson, L. y Lepore, E. (2013). Slurring words. Noûs, 47(1), 25-48.

Barbujani, G. (2016). El lugar de la raza. En M. Aime, (Ed.), Contra el racismo (pp. 25-72). Barcelona: Editorial Libros.

Bruner, J. (1981). Realidad Mental y mundos posibles. Barcelona: Gedisa.

Butler, J. (1997). Excitable Speech. A Politic of the Performative. New York: Routledge.

Butler, J. (2016). Los sentidos del sujeto. Barcelona: Herder.

Cisneros, J. (2008). Contaminated communities: The metaphor of "immigrant as pollutant" in media representations of immigration. Rhetoric $\mathcal{E}$ public affairs, 11(4), 569-601.

Delgado, R. (1982). Words that wound. A tord action for racial insults, epithets and name-calling. Harvard Civil Rights-Civil Liberties Law Review, 17, 133-181.

Enck-Wanzer, D. (2011). Barack Obama, the Tea Party, and the threat of race: On racial neoliberalism and born again racism. Communication, Culture and Critique, 4(1), 23-30.

Faloppa, F. (2016). Por un lenguaje no racista. En M. Aime, (Ed.), Contra el racismo (pp. 105-178). Barcelona: Editorial Libros.

Geertz, C. (1993). La interpretación de la cultura. Barcelona: Gedisa.

Giroux, H. (2013). Una pedagogía de la resistencia en la edad del capitalismo de casino. Con-ciencia social, 17, 55-71.

Howarth, C. (2002). Identity in whose eyes? The role of representations in identity construction. Journal for the theory of social behaviour, 32(2), 145-162.

Jungkunz, V. y White, J. (2013). Ignorance, innocence, and democratic responsibility: Seeing race, hearing racism. The Journal of Politics, 75(2), 436-450

Quijano, A. (1999). iQué tal raza! Recuperado de: https://antropologiadeoutraforma.files.wordpress. com/2013/04/quijano-anibal-que-tal-raza.pdf

Labov, W. y Waletzky, J. (1967), "Narrative analysis: oral versions of personal experience", En J. Helm, Essays on the Verbal and Visual Arts (pp.12-44). Seattle: University of Washington Press.

Lakoff, G. y Johnson, M. (1991). Metáforas de la vida cotidiana. Madrid: Cátedra.

Lewontin, R. (1972). The apportionment of human diversity. Evolutionary Biology, 6, 381-98.

Livingstone, F. (1962). On the nonexistence of human races. Current anthropology, 3, 279-81.
Maalouf, A. (2016). Identidades asesinas. Madrid: Alianza Editorial.

Martín, L. (2006). El análisis crítico del discurso. Fronteras y exclusión social en los discursos racistas. En Análisis del discurso. Manual para las ciencias sociales (pp. 161-196). Barcelona: Editorial UOC.

Martín, L. y Van Dijk, T. (1988). "Había un problema y se ha solucionado". Legitimación de la expulsión de inmigrantes "ilegales" en el discurso parlamentarios español. En L. Martín y R. Whittaker, Poder-Decir o el poder de los discursos (pp. 169- 234). Madrid: Arrecife.

Mbembe, A. (2016). Crítica de la razón negra. Barcelona: NED.

Mignolo, W. (2003). Historias locales/diseños globales. Colonialidad, conocimientos subalternos $y$ pensamiento fronterizo. Madrid: Akal.

Oka, K. (2005). Racism "renewed". En L. Karumanchery (Ed.), Engaging Equity (pp. 27-40). Alberta: Detseling Enterprises.

Polletta, F. (2006). It was like a fever. Storytelling in protest and politics. Chicago: The University of Chicago Press

Reisigl, M., \& Wodak, R. (2005). Discourse and discrimination: Rhetoric's of racism and antisemitism. New York: Routledge.

Skliar, C. y Duschatzky, S. (2015). La diversidad bajo sospecha. Recuperado de: http:/www. porlainclusionmercosur.educ.ar/documentos/ Ladiversidadbajosospecha.pdf

Skliar, C. (2010). La experiencia de la conversación, de la mirada y de la investigación educativa. En N. Pérez. y J. Contreras (Comps.), Investigar la experiencia educativa (pp. 136-152). Madrid: Morata.

Soler, S. y Pardo, N. (2007). Discurso y racismo en Colombia: cinco siglos de invisibilidad y exclusión. En T. Van Dijk (Coord.), Racismo y discurso en América Latina (pp. 181-228). Barcelona: Gedisa.

Soler, S. (2008). Racismo y discurso en los textos escolares. Representación de la diversidad étnica y racial en los textos de ciencias sociales en Colombia. En J. Arocha (ed.), Nina S. de Friedemann: cronista de disidencias y resistencias (pp. 233-265). Bogotá: Universidad Nacional de Colombia.

Soler, S. (2011). Análisis crítico del discurso de documentos de política pública en educación. Forma y Función, 24(1), 75-105.

Soler, S. (2013). Entre negro oscuro y moreno claro. Discursos e identidades étnicas de niños y niñas afrodescendiente en contexto escolar en Bogotá. Educar em Revista, 47, 111-143. 
Soler, S. (2018). The Indigenous People in the General Laws of Education of Colombia. An approach from the Critical Discourse Analysis Perspective. Wulfenia, 25(6), 13-35.

Steinberg, S. (2005). The dialectics of power. En L. Karumanchery (Ed.), Engaging Equity (pp. 13-26). Alberta: Detseling Enterprises Lta.

Taylor, P. (2013). Race. A Philosophical introduction. Cambridge: Polity Press.

Van Dijk, T. (2015). Critical discourse studies: A sociocognitive approach. En R. Wodak y M. Meyer (Eds.), Methods of critical discourse studies (pp 6374). London: Sage.

Van Dijk, T. (Comp.). (2000). El discurso como interacción social. Barcelona: Gedisa.

Van Dijk, T. (1997). Historias y racismo. En D. Mumby (Comp.), Narrativa y control social. Perspectivas críticas (pp. 163-190). Buenos Aires: Amarrortu Editores.
Van Dijk, T. (1993). Elite discourse and racism. Newbury Park: Sage.

Van Leeuwen, T. (1996). Social actor representation. En C. Caldas-Coulthard y M. Coulthard (Eds.) Texts and Practices. Readings in Critical Discourse Analysis. Londres: Routhledge.

Vargas, P. (2016). Los indígenas en las Crónicas de indias: una aproximación a su comprensión desde los estudios críticos. [Tesis doctoral]. Bogotá, Universidad distrital Francisco José de Caldas.

Welch, K. (2007). Black criminal stereotypes and racial profiling. Journal of Contemporary Criminal Justice, 23(3), 276-288

Wetherell, M. y Potter, J. (1993). Mapping the language of racism: Discourse and the legitimation of exploitation. Columbia: Columbia University Press. 